\title{
Anti-periodic boundary value problems of fractional differential equations with the Riemann-Liouville fractional derivative
}

\section{Guoqing Chai*}

\section{"Correspondence:}

mathchgq@gmail.com

College of Mathematics and

Statistics, Hubei Normal University,

Hubei, 435002, P.R. China

\begin{abstract}
In this paper, the author puts forward a kind of anti-periodic boundary value problems of fractional equations with the Riemann-Liouville fractional derivative. More precisely, the author is concerned with the following fractional equation:

$$
D_{0+}^{\alpha} u(t)=f\left(t, u(t), u^{\prime}(t)\right), \quad t \in(0,1)
$$

with the anti-periodic boundary value conditions$$
t^{2-\alpha} u(t)_{\mid t \rightarrow 0^{+}}=-t^{2-\alpha} u(t)_{\mid t=1}, \quad\left(t^{2-\alpha} u(t)\right)_{\mid t \rightarrow 0^{+}}^{\prime}=-\left(t^{2-\alpha} u(t)\right)_{\mid t=1}^{\prime},
$$

where $D_{0+}^{\alpha}$ denotes the standard Riemann-Liouville fractional derivative of order $\alpha \in(1,2)$, and the nonlinear function $f(t, \cdot, \cdot)$ may be singular at $t=0$. By applying the contraction mapping principle and the other fixed point theorem, the author obtains the existence and uniqueness of solutions.
\end{abstract}

MSC: $34 \mathrm{~A} 08 ; 34 \mathrm{~B} 15$

Keywords: fractional differential equations; anti-periodic boundary value problems; existence results; fixed point theorem

\section{Introduction}

In the present paper, we are concerned with the existence of solutions for the fractional differential equation

$$
D_{0+}^{\alpha} u(t)=f\left(t, u(t), u^{\prime}(t)\right), \quad t \in(0,1)
$$

with anti-periodic boundary value conditions

$$
t^{2-\alpha} u(t)_{\mid t \rightarrow 0^{+}}=-t^{2-\alpha} u(t)_{\mid t=1}, \quad\left(t^{2-\alpha} u(t)\right)_{\mid t \rightarrow 0^{+}}^{\prime}=-\left(t^{2-\alpha} u(t)\right)_{\mid t=1}^{\prime},
$$

where $D_{0_{+}}^{\alpha}$ denotes the standard Riemann-Liouville fractional derivative of order $\alpha \in(1,2)$, and the nonlinear function $f(t, \cdot, \cdot)$ may be singular at $t=0$.

Differential equations with fractional order are a generalization of ordinary differential equations to non-integer order. This generalization is not a mere mathematical curiosity but rather has interesting applications in many areas of science and engineering such

\section{Springer}

○2013 Chai; licensee Springer. This is an Open Access article distributed under the terms of the Creative Commons Attribution License (http://creativecommons.org/licenses/by/2.0), which permits unrestricted use, distribution, and reproduction in any medium, provided the original work is properly cited. 
as electrochemistry, control, porous media, electromagnetism, etc. (see [1-4]). There has been a significant development in the study of fractional differential equations in recent years; see, for example, [5-21].

Anti-periodic boundary value problems occur in mathematic modeling of a variety of physical processes and have recently received considerable attention. For examples and details of anti-periodic fractional boundary value problems, see [22-31] and the references therein. However, up to now, almost all studies on anti-periodic fractional boundary value problems have been devoted to fractional equations with the Caputo fractional derivative. Recently, there appeared a paper dealing with anti-periodic boundary value problems of a fractional equation with the Riemann-Liouville fractional derivative (see [22]), which will be formulated later. The main reason is that in general the Riemann-Liouville fractional derivative $u^{(i)}(0)(0 \leq i<\alpha)$ does not exist unless $u^{(i)}(0)=0$, and therefore there is some difficulty in expressing anti-periodic boundary value conditions with the RiemannLiouville fractional derivative. So, no matter which kind of anti-periodic boundary value conditions we shall propose, first of all, we must ensure that the limits exist taken on the left-hand side of the formula on the anti-periodic boundary value conditions when variable tends to zero. In the paper [22] mentioned above, Ahmad and Nieto put forward a kind of boundary value problems as follows:

$$
\left\{\begin{array}{l}
D^{\alpha} u(t)=f(t, u(t)), \quad t \in[0, T], 0<\alpha \leq 2, \\
I_{0+}^{2-\alpha} u\left(0^{+}\right)=b_{0} I_{0+}^{2-\alpha} u\left(T^{-}\right), \quad D_{0+}^{\alpha-1} u\left(0^{+}\right)=b_{1} D_{0+}^{\alpha-1} u\left(T^{-}\right), \quad b_{0} \neq 1, b_{1} \neq 1 .
\end{array}\right.
$$

It is well known that the limits $I_{0+}^{2-\alpha} u\left(0^{+}\right)$and $D_{0+}^{\alpha-1} u\left(0^{+}\right)$exist. Moreover, it can be verified that $t^{2-\alpha} u(t)_{\mid t \rightarrow 0^{+}}=\frac{1}{\Gamma(\alpha-1)} I_{0+}^{2-\alpha} u\left(0^{+}\right),\left(t^{2-\alpha} u(t)\right)_{\mid t \rightarrow 0^{+}}^{\prime}=\frac{1}{\Gamma(\alpha)} D_{0+}^{\alpha-1} u\left(0^{+}\right)$. Especially, comparing with the recent article (see [32]) dealing with the following periodic boundary value problems with Riemann-Liouville fractional derivative

$$
\left\{\begin{array}{l}
D^{2 \alpha} u(t)=f\left(t, u, D^{\alpha} u\right), \quad t \in(0,1] \backslash\left\{t_{1}, t_{2}, \ldots, t_{m}\right\}, 0<\alpha \leq 1, \\
\left.t^{1-\alpha} u(t)\right|_{t \rightarrow 0+}=u(1),\left.\quad t^{1-\alpha} D^{\alpha} u(t)\right|_{t \rightarrow 0}=D^{\alpha} u(1), \\
\left.\left(t-t_{j}\right)^{1-\alpha}\left(u(t)-u\left(t_{j}\right)\right)\right|_{t \rightarrow t_{j}^{+}}=I_{j}\left(u\left(t_{j}\right)\right), \\
\left.\left(t-t_{j}\right)^{1-\alpha}\left(D^{\alpha} u(t)-D^{\alpha} u\left(t_{j}\right)\right)\right|_{t \rightarrow t_{j}^{+}}=\bar{I}_{j}\left(u\left(t_{j}\right)\right),
\end{array}\right.
$$

and taking into account the consistency with integer order anti-periodic boundary value problems, we consider the anti-periodic boundary value condition (1.2) in the present paper to be more natural and suitable. It is noteworthy that such a form of anti-periodic boundary value conditions (1.2) is very convenient to construct an appropriate Banach space which coordinates the feature of the solution $u$ because of the fact that $\lim u(t)=\infty$ $\left(t \rightarrow 0^{+}\right)$may occur and function $f(t, \cdot, \cdot)$ may be singular at $t=0$. Moreover, when $\alpha \rightarrow 2$ in (1.2), the anti-periodic boundary value conditions in (1.2) are changed into $u(0)=-u(1)$, $u^{\prime}(0)=-u^{\prime}(1)$, which are coincident with anti-periodic boundary value conditions of second-order differential equations (see [33]).

The rest of this paper is organized as follows. In Section 2, we present some necessary definitions and preliminary results that will be used to prove our main results. In Section 3, we put forward and prove our main results. By applying the contraction mapping principle and the other fixed point theorem, we obtain the existence and uniqueness of solutions for boundary value problems (simply denoted by BVP). Finally, we give two examples to demonstrate our main results. 


\section{Preliminaries}

In this section, we introduce some preliminary facts which are used throughout this paper. Let $\mathbb{N}$ be the set of positive integers, $\mathbb{R}$ be the set of real numbers.

Definition 2.1 ([3]) The Riemann-Liouville fractional integral of order $\alpha>0$ of a function $y:(a, b] \rightarrow \mathbb{R}$ is given by

$$
I_{a+}^{\alpha} y(t)=\frac{1}{\Gamma(\alpha)} \int_{0}^{t}(t-s)^{\alpha-1} y(s) d s, \quad t \in(a, b] .
$$

Definition 2.2 ([3]) The Riemann-Liouville fractional derivative of order $\alpha>0$ of a function $y:(a, b] \rightarrow \mathbb{R}$ is given by

$$
D_{a+}^{\alpha} y(t)=\frac{1}{\Gamma(n-\alpha)}\left(\frac{d}{d t}\right)^{n} \int_{0}^{t} \frac{y(s)}{(t-s)^{\alpha-n+1}} d s, \quad t \in(a, b],
$$

where $n=[\alpha]+1,[\alpha]$ denotes the integer part of $\alpha$.

Lemma 2.1 ([34] Let $\alpha>0$. If $u \in C(0,1) \cap L(0,1)$ with a fractional derivative of order $\alpha$ that belongs to $C(0,1) \cap L(0,1)$, then

$$
I_{0+}^{\alpha} D_{0+}^{\alpha} u(t)=u(t)+c_{1} t^{\alpha-1}+c_{2} t^{\alpha-2}+\cdots+c_{n} t^{\alpha-n}
$$

for some $c_{i} \in \mathbb{R}, i=1,2, \ldots, n$, where $n=[\alpha]+1$.

Let

$$
\begin{aligned}
& X_{1}=\left\{u: u \in C^{\prime}(0,1], \lim _{t \rightarrow 0^{+}} t^{2-\alpha} u(t) \text { and } \lim _{t \rightarrow 0^{+}}\left(t^{2-\alpha} u(t)\right)^{\prime} \text { exist }\right\}, \quad \text { and } \\
& X_{0}=\left\{u: u \in C(0,1] \cap L(0,1), \sup _{t \in(0,1]}\left|t^{2-\alpha} u(t)\right|<\infty\right\} .
\end{aligned}
$$

It is easy to verify that $X_{1}$ is a normed linear space with the norm

$$
\|u\|_{1}=\max \left\{\sup _{t \in(0,1]}\left|t^{2-\alpha} u(t)\right|, \sup _{t \in(0,1]}\left|\left(t^{2-\alpha} u(t)\right)^{\prime}\right|\right\}, \quad u \in X_{1},
$$

and $X_{0}$ is a normed linear space with the norm

$$
\|u\|_{0}=\sup _{t \in(0,1]}\left|t^{2-\alpha} u(t)\right|, \quad u \in X_{0}
$$

respectively. Moreover, we have the following lemma.

Lemma 2.2 $X_{1}$ is a Banach space with the norm $\|u\|_{1}$.

Proof In fact, let $\left\{u_{n}\right\}$ be any sequence in $X_{1}$ with $\left\|u_{n}-u_{m}\right\|_{1} \rightarrow 0$. Then, for arbitrary $\varepsilon>0$, there exists $N \in \mathbb{N}$ such that

$$
\left\|u_{n}-u_{m}\right\|_{1}<\varepsilon
$$


when $n, m \geq N$. So,

$$
\begin{aligned}
& \left|t^{2-\alpha} u_{n}(t)-t^{2-\alpha} u_{m}(t)\right|<\varepsilon, \quad t \in(0,1], \\
& \left|\left(t^{2-\alpha} u_{n}(t)\right)^{\prime}-\left(t^{2-\alpha} u_{m}(t)\right)^{\prime}\right|<\varepsilon, \quad t \in(0,1],
\end{aligned}
$$

when $n, m \geq N$.

In view of (2.2), there exists $v \in C(0,1]$ such that $t^{2-\alpha} u_{n} \rightarrow v$ uniformly on $(0,1]$, and so

$$
u_{n}(t) \rightarrow v(t) t^{\alpha-2}, \quad t \in(0,1]
$$

Set $u=v(t) t^{\alpha-2}$. Then $u_{n}(t) \rightarrow u(t), t \in(0,1]$ and $u \in C(0,1]$. So, letting $m \rightarrow+\infty$ in (2.2), we have

$$
\left|t^{2-\alpha} u_{n}(t)-t^{2-\alpha} u(t)\right| \leq \varepsilon, \quad t \in(0,1]
$$

when $n \geq N$. That is, $t^{2-\alpha} u_{n} \rightarrow t^{2-\alpha} u$ uniformly on $(0,1]$. Because $\lim _{t \rightarrow 0^{+}} t^{2-\alpha} u_{n}(t)$ exists for all $n \in \mathbb{N}$, by applying the theorem to the limit convergence of function sequences, we know that $\lim _{n \rightarrow \infty} \lim _{t \rightarrow 0^{+}} t^{2-\alpha} u_{n}(t)$ exists, and

$$
\lim _{t \rightarrow 0^{+}} t^{2-\alpha} u(t)=\lim _{t \rightarrow 0^{+}} \lim _{n \rightarrow \infty} t^{2-\alpha} u_{n}(t)=\lim _{n \rightarrow \infty} \lim _{t \rightarrow 0^{+}} t^{2-\alpha} u_{n}(t) .
$$

So, $\lim _{t \rightarrow 0^{+}} t^{2-\alpha} u(t)$ exists.

Similarly, applying the theorem to the differentiability of function sequences and letting $m \rightarrow \infty$ in (2.3), we obtain

$$
\left|\left(t^{2-\alpha} u_{n}(t)\right)^{\prime}-\left(t^{2-\alpha} u(t)\right)^{\prime}\right|<\varepsilon, \quad t \in(0,1]
$$

whenever $n \geq N$.

Let $f_{n}(t)=\left(t^{2-\alpha} u_{n}(t)\right)^{\prime}$ and $f(t)=\left(t^{2-\alpha} u(t)\right)^{\prime}$. Then (2.4) means that $f_{n} \rightarrow f$ uniformly on (0.1], and so

$$
\lim _{t \rightarrow 0^{+}} f(t)=\lim _{n \rightarrow \infty} \lim _{t \rightarrow 0^{+}} f_{n}(t) \quad \text { exists }
$$

by applying the theorem to the limit convergence of function sequences again. That is,

$$
\lim _{t \rightarrow 0^{+}}\left(t^{2-\alpha} u(t)\right)^{\prime}=\lim _{n \rightarrow \infty} \lim _{t \rightarrow 0^{+}}\left(t^{2-\alpha} u_{n}(t)\right)^{\prime} \quad \text { exists. }
$$

Thus, $\lim _{t \rightarrow 0^{+}}\left(t^{2-\alpha} u(t)\right)^{\prime}$ exists.

To summarize, $\left\|u_{n}-u\right\|_{1} \rightarrow 0$ with $u \in X_{1}$.

Now, we consider the following auxiliary boundary value problem:

$$
\left\{\begin{array}{l}
D_{0+}^{\alpha} u(t)=h(t), \quad t \in(0,1], \\
t^{2-\alpha} u(t)_{\mid t \rightarrow 0^{+}}=-t^{2-\alpha} u(t)_{\mid t=1}, \\
\left(t^{2-\alpha} u(t)\right)_{\mid t \rightarrow 0^{+}}^{\prime}=-\left(t^{2-\alpha} u(t)\right)_{\mid t=1}^{\prime},
\end{array}\right.
$$

where $h \in X_{0}$. 
We have the following lemma.

Lemma 2.3 For a given $h \in X_{0}$, if $u \in X_{1}$ is a solution of $B V P(2.5)$, then $u$ is given by

$$
\begin{aligned}
u(t)= & -t^{\alpha-1} \int_{0}^{1} G_{1}(s) h(s) d s+t^{\alpha-2} \int_{0}^{1} G_{2}(s) h(s) d s \\
& +\frac{1}{\Gamma(\alpha)} \int_{0}^{t}(t-s)^{\alpha-1} h(s) d s, \quad t \in(0,1]
\end{aligned}
$$

where

$$
\begin{aligned}
& G_{1}(s)=\frac{1}{2 \Gamma(\alpha)}\left[(2-\alpha)(1-s)^{\alpha-1}+(\alpha-1)(1-s)^{\alpha-2}\right], \quad s \in(0,1), \\
& G_{2}(s)=\frac{1}{4 \Gamma(\alpha)}\left[(\alpha-1)(1-s)^{\alpha-2}-\alpha(1-s)^{\alpha-1}\right], \quad s \in(0,1) .
\end{aligned}
$$

Proof In fact, owing to the fact that $h \in X_{0}$, in view of Lemma 2.1, we have

$$
u(t)=c_{1} t^{\alpha-1}+c_{2} t^{\alpha-2}+I_{0+}^{\alpha} h(t), \quad t \in(0,1]
$$

for some $c_{1}, c_{2} \in \mathbb{R}$.

So,

$$
t^{2-\alpha} u(t)=c_{1} t+c_{2}+t^{2-\alpha} I_{0+}^{\alpha} h(t), \quad t \in(0,1]
$$

and

$$
\left(t^{2-\alpha} u(t)\right)^{\prime}=c_{1}+(2-\alpha) t^{1-\alpha} I_{0+}^{\alpha} h(t)+t^{2-\alpha} I_{0+}^{\alpha-1} h(t), \quad t \in(0,1] .
$$

Because $\left|s^{2-\alpha} h(s)\right| \leq\|h\|_{0}, s \in(0,1]$, we have

$$
\begin{aligned}
\left|I_{0+}^{\alpha} h(t)\right| & \leq \frac{1}{\Gamma(\alpha)} \int_{0}^{t}(t-s)^{\alpha-1}|h(s)| d s \\
& =\frac{1}{\Gamma(\alpha)} \int_{0}^{t}(t-s)^{\alpha-1} s^{\alpha-2}\left|s^{2-\alpha} h(s)\right| d s \\
& \leq \frac{\|h\|_{0}}{\Gamma(\alpha)} \int_{0}^{t}(t-s)^{\alpha-1} s^{\alpha-2} d s \\
& \stackrel{s=t \tau}{=} \frac{\|h\|_{0}}{\Gamma(\alpha)} t^{2(\alpha-1)} \int_{0}^{1}(1-\tau)^{\alpha-1} \tau^{\alpha-2} d \tau \\
& =\frac{\Gamma(\alpha-1)}{\Gamma(2 \alpha-1)}\|h\|_{0} t^{2(\alpha-1)}, \quad t \in(0,1] .
\end{aligned}
$$

Thus,

$$
\begin{aligned}
\left|t^{2-\alpha} I_{0+}^{\alpha} h(t)\right| & \leq \frac{\Gamma(\alpha-1)}{\Gamma(2 \alpha-1)}\|h\|_{0} t^{\alpha}, \\
\left|t^{1-\alpha} I_{0+}^{\alpha} h(t)\right| & \leq \frac{\Gamma(\alpha-1)}{\Gamma(2 \alpha-1)}\|h\|_{0} t^{\alpha-1}, \quad t \in(0,1] .
\end{aligned}
$$


Similarly, we have

$$
\left|t^{2-\alpha} I_{0+}^{\alpha-1} h(t)\right| \leq \frac{\Gamma(\alpha-1)}{\Gamma(2 \alpha-2)}\|h\|_{0} t^{\alpha-1}, \quad t \in(0,1]
$$

From (2.13)-(2.14), it follows that

$$
\begin{aligned}
& \lim _{t \rightarrow 0+} t^{2-\alpha} I_{0+}^{\alpha} h(t)=0, \\
& \lim _{t \rightarrow 0+} t^{1-\alpha} I_{0+}^{\alpha} h(t)=0,
\end{aligned}
$$

and

$$
\lim _{t \rightarrow 0+} t^{2-\alpha} I_{0+}^{\alpha-1} h(t)=0
$$

So, by the boundary value conditions in (2.5) together with (2.10)-(2.11), from (2.15)-(2.17) it follows that

$$
\begin{aligned}
& c_{1}+2 c_{2}+I_{0+}^{\alpha} h(1)=0, \\
& 2 c_{1}+(2-\alpha) I_{0+}^{\alpha} h(1)+I_{0+}^{\alpha-1} h(1)=0 .
\end{aligned}
$$

Thus, (2.18)-(2.19) imply that

$$
\begin{aligned}
& c_{1}=-\frac{2-\alpha}{2} I_{0+}^{\alpha} h(1)-\frac{1}{2} I_{0+}^{\alpha-1} h(1), \\
& c_{2}=-\frac{\alpha}{4} I_{0+}^{\alpha} h(1)+\frac{1}{4} I_{0+}^{\alpha-1} h(1) .
\end{aligned}
$$

Substituting (2.20)-(2.21) into (2.9), we have

$$
\begin{aligned}
u(t) & =-\frac{1}{2}\left[(2-\alpha) I_{0+}^{\alpha} h(1)+I_{0+}^{\alpha-1} h(1)\right] t^{\alpha-1}+\frac{1}{4}\left[I_{0+}^{\alpha-1} h(1)-\alpha I_{0+}^{\alpha} h(1)\right] t^{\alpha-2}+I_{0+}^{\alpha} h(t) \\
& =-t^{\alpha-1} \int_{0}^{1} G_{1}(s) h(s) d s+t^{\alpha-2} \int_{0}^{1} G_{2}(s) h(s) d s+I_{0+}^{\alpha} h(t), \quad t \in(0,1],
\end{aligned}
$$

where $G_{1}, G_{2}$ are given by (2.7) and (2.8), respectively.

The proof is complete.

We definite an operator $T$ on $X_{0}$ as follows:

$$
\operatorname{Th}(t)=-t^{\alpha-1} \int_{0}^{1} G_{1}(s) h(s) d s+t^{\alpha-2} \int_{0}^{1} G_{2}(s) h(s) d s+I_{0+}^{\alpha} h(t), \quad t \in(0,1]
$$

for $h \in X_{0}$.

We first establish the following lemma.

Lemma 2.4 $T X_{0} \subset X_{1}$. 
Proof For any $h \in X_{0}$, from (2.12), we have that

$$
\int_{0}^{1}(1-s)^{\alpha-1}|h(s)| d s \leq \frac{\Gamma(\alpha) \Gamma(\alpha-1)}{\Gamma(2 \alpha-1)}\|h\|_{0} .
$$

Again,

$$
\begin{aligned}
\int_{0}^{1}(1-s)^{\alpha-2}|h(s)| d s & =\int_{0}^{1}(1-s)^{\alpha-2} s^{\alpha-2}\left|s^{2-\alpha} h(s)\right| d s \\
& \leq\|h\|_{0} \int_{0}^{1}(1-s)^{\alpha-2} s^{\alpha-2} d s \\
& =\|h\|_{0} B(\alpha-1, \alpha-1) .
\end{aligned}
$$

Thus, from (2.7)-(2.8), we know that the integrals $\int_{0}^{1} G_{1}(s) h(s) d s$ and $\int_{0}^{1} G_{2}(s) h(s) d s$ converge. So, from (2.12) and (2.22), it follows that $T h(t)$ exists on $(0,1]$. That is, the operator $T$ is well defined.

In what follows, we show that $T h \in X_{1}$.

From $h \in X_{0}$, we know that $h \in C(0,1]$ and $\left|s^{2-\alpha} h(s)\right| \leq\|h\|_{0}$. Let $\phi(s)=s^{2-\alpha} h(s), s \in(0,1]$. Then $\phi \in C(0,1]$ and $|\phi(s)| \leq\|h\|_{0}, s \in(0,1]$, and so $\psi \in C((0,1] \times(0,1])$ and $|\psi(t, s)| \leq$ $\|h\|_{0}$ by setting $\psi(t, \tau)=\phi(t \tau),(t, \tau) \in(0,1] \times(0,1]$.

Since

$$
\begin{aligned}
\int_{0}^{t}(t-s)^{\alpha-2} h(s) d s & =\int_{0}^{t}(t-s)^{\alpha-2} s^{2-\alpha} \phi(s) d s \\
& \stackrel{s=t \tau}{=} t^{2 \alpha-3} \int_{0}^{1}(1-\tau)^{\alpha-2} \tau^{\alpha-2} \psi(t, \tau) d \tau,
\end{aligned}
$$

and $|\psi(t, \tau)| \leq\|h\|_{0}, \psi \in C((0,1] \times(0,1])$, we know that $\int_{0}^{t}(t-s)^{\alpha-2} h(s) d s$ is continuous on $(0,1]$ from $(2.23)$ and applying the Lebesgue convergence theorem. Consequently, $(T h)^{\prime} \in C(0,1]$ according to

$$
\begin{aligned}
(T h)^{\prime}(t)= & -(\alpha-1) t^{\alpha-2} \int_{0}^{1} G_{1}(s) h(s) d s+(\alpha-2) t^{\alpha-3} \int_{0}^{1} G_{2}(s) h(s) d s \\
& +\frac{1}{\Gamma(\alpha-1)} \int_{0}^{t}(t-s)^{\alpha-2} h(s) d s, \quad t \in(0,1]
\end{aligned}
$$

by (2.22).

Finally, in view of (2.22), we have

$$
t^{2-\alpha}(T h)(t)=-t \int_{0}^{1} G_{1}(s) h(s) d s+\int_{0}^{1} G_{2}(s) h(s) d s+t^{2-\alpha} I_{0+}^{\alpha} h(t), \quad t \in(0,1]
$$

and

$$
\begin{aligned}
\left(t^{2-\alpha}(T h)(t)\right)^{\prime}= & -\int_{0}^{1} G_{1}(s) h(s) d s+(2-\alpha) t^{1-\alpha} I_{0+}^{\alpha} h(t) \\
& +\frac{1}{\Gamma(\alpha-1)} t^{2-\alpha} \int_{0}^{t}(t-s)^{\alpha-2} h(s) d s, \quad t \in(0,1] .
\end{aligned}
$$


Again,

$$
\begin{aligned}
\frac{t^{2-\alpha}}{\Gamma(\alpha-1)}\left|\int_{0}^{t}(t-s)^{\alpha-2} h(s) d s\right| & \leq \frac{\|h\|_{0} t^{2-\alpha}}{\Gamma(\alpha-1)} \int_{0}^{t}(t-s)^{\alpha-2} s^{\alpha-2} d s \\
& \stackrel{s=t \tau}{=} \frac{\|h\|_{0} t^{\alpha-1}}{\Gamma(\alpha-1)} \int_{0}^{1}(1-\tau)^{\alpha-2} \tau^{\alpha-2} d \tau \\
& =\frac{\Gamma(\alpha-1)\|h\|_{0}}{\Gamma(2 \alpha-2)} t^{\alpha-1}, \quad t \in(0,1] .
\end{aligned}
$$

Hence, formula (2.25) together with (2.13) implies that $\lim _{t \rightarrow 0^{+}} t^{2-\alpha}(T h)(t)=\int_{0}^{1} G_{2}(s) d s$ exists, and formula (2.26) together with (2.13)-(2.27) implies that $\lim _{t \rightarrow 0^{+}}\left(t^{2-\alpha}(T h)(t)\right)^{\prime}=$ $-\int_{0}^{1} G_{1}(s) d s$ exists.

Summing up the above analysis, we obtain that $T h \in X_{1}$. The proof is complete.

We need the following lemma, which is important in establishing our main result in the next section.

Lemma 2.5 $T: X_{0} \rightarrow X_{1}$ is completely continuous.

Proof We divide the proof into two parts.

Part 1. First, we show that $T$ is a continuous operator.

Let $\left\{u_{n}\right\}$ be an arbitrary sequence in $X_{0}$ with $u_{n} \rightarrow u \in X_{0}$. Then

$$
\left|s^{2-\alpha}\left(u_{n}(s)-u(s)\right)\right| \leq\left\|u_{n}-u\right\|_{0}, \quad s \in(0,1] .
$$

Thus, from (2.25), it follows that

$$
\begin{aligned}
t^{2-\alpha}\left|T u_{n}(t)-T u(t)\right| \leq & t \int_{0}^{1} G_{1}(s)\left|u_{n}(s)-u(s)\right| d s+\int_{0}^{1}\left|G_{2}(s)\right| \cdot\left|u_{n}(s)-u(s)\right| d s \\
& +\frac{t^{2-\alpha}}{\Gamma(\alpha)} \int_{0}^{t}(t-s)^{\alpha-1}\left|u_{n}(s)-u(s)\right| d s \\
\leq & {\left[t \int_{0}^{1} G_{1}(s) s^{\alpha-2} d s+\int_{0}^{1}\left|G_{2}(s)\right| s^{\alpha-2} d s\right.} \\
& \left.+\frac{t^{2-\alpha}}{\Gamma(\alpha)} \int_{0}^{t}(t-s)^{\alpha-1} s^{\alpha-2} d s\right] \cdot\left\|u_{n}-u\right\|_{0} \\
\leq & \frac{(5 \alpha+2) \Gamma(\alpha-1)}{4 \Gamma(2 \alpha-1)}\left\|u_{n}-u\right\|_{0}, \quad t \in(0,1],
\end{aligned}
$$

noting that

$$
\int_{0}^{1} G_{1}(s) s^{\alpha-2} d s=\frac{\alpha \Gamma(\alpha-1)}{2 \Gamma(2 \alpha-1)}, \quad \int_{0}^{1}\left|G_{2}(s)\right| s^{\alpha-2} d s \leq \frac{(3 \alpha-2) \Gamma(\alpha-1)}{4 \Gamma(2 \alpha-1)},
$$

and

$$
\begin{aligned}
\frac{1}{\Gamma(\alpha)} \int_{0}^{t}(t-s)^{\alpha-1} s^{\alpha-2} d s & \stackrel{s=t \tau}{=} \frac{t^{2(\alpha-1)}}{\Gamma(\alpha)} \int_{0}^{1}(1-\tau)^{\alpha-1} \tau^{\alpha-2} d \tau \\
& =\frac{\Gamma(\alpha-1)}{\Gamma(2 \alpha-1)} t^{2(\alpha-1)}, \quad t \in(0,1]
\end{aligned}
$$


So,

$$
\sup _{t \in(0,1]} t^{2-\alpha}\left|T u_{n}(t)-T u(t)\right| \leq \frac{(5 \alpha+2) \Gamma(\alpha-1)}{4 \Gamma(2 \alpha-1)}\left\|u_{n}-u\right\|_{0} .
$$

Similarly, from (2.26), (2.28) and (2.29), we have

$$
\begin{aligned}
\mid\left(t^{2-\alpha}\left(\left(T u_{n}\right)(t)-(T u)(t)\right)^{\prime} \mid \leq\right. & {\left[\int_{0}^{1} G_{1}(s) s^{\alpha-2} d s+\frac{2-\alpha}{\Gamma(\alpha)} t^{1-\alpha} \int_{0}^{t}(t-s)^{\alpha-1} s^{\alpha-2} d s\right.} \\
& \left.+\frac{1}{\Gamma(\alpha-1)} t^{2-\alpha} \int_{0}^{t}(t-s)^{\alpha-2} s^{\alpha-2} d s\right]\left\|u_{n}-u\right\|_{0} \\
\leq & \frac{3 \alpha \Gamma(\alpha-1)}{2 \Gamma(2 \alpha-1)}\left\|u_{n}-u\right\|_{0}, \quad t \in(0,1]
\end{aligned}
$$

noting that

$$
\begin{aligned}
\frac{1}{\Gamma(\alpha-1)} \int_{0}^{t}(t-s)^{\alpha-2} s^{\alpha-2} d s & \stackrel{s=t \tau}{=} \frac{t^{2 \alpha-3}}{\Gamma(\alpha-1)} \int_{0}^{1}(1-\tau)^{\alpha-2} \tau^{\alpha-2} d \tau \\
& =\frac{\Gamma(\alpha-1)}{\Gamma(2 \alpha-2)} t^{2 \alpha-3}, \quad t \in(0,1] .
\end{aligned}
$$

So,

$$
\sup _{t \in(0,1]} \mid\left(t^{2-\alpha}\left(T u_{n}(t)-T u(t)\right)^{\prime} \mid \leq \frac{3 \alpha \Gamma(\alpha-1)}{2 \Gamma(2 \alpha-1)}\left\|u_{n}-u\right\|_{0} .\right.
$$

From (2.30)-(2.31), we have that

$$
\left\|T u_{n}-T u\right\|_{1} \leq D\left\|u_{n}-u\right\|_{0}
$$

where

$$
D=\max \left\{\frac{(5 \alpha+2) \Gamma(\alpha-1)}{4 \Gamma(2 \alpha-1)}, \frac{3 \alpha \Gamma(\alpha-1)}{2 \Gamma(2 \alpha-1)}\right\}=\frac{(5 \alpha+2) \Gamma(\alpha-1)}{4 \Gamma(2 \alpha-1)} .
$$

So, $T$ is a continuous operator on $X_{0}$.

Part 2. Now we show that $T$ is a compact operator.

Assume that $\Omega$ is an arbitrary bounded set in $X_{0}$. Then there exists $M>0$ such that $\|u\|_{0} \leq M$ for all $u \in \Omega$. By an argument similar to (2.30) and (2.32) in Part 1, from (2.25)(2.26), we can obtain

$$
\begin{aligned}
& \sup _{t \in(0,1]}\left|t^{2-\alpha} T u(t)\right| \leq \frac{(5 \alpha+2) \Gamma(\alpha-1)}{4 \Gamma(2 \alpha-1)}\|u\|_{0}, \\
& \sup _{t \in(0,1]}\left|\left(t^{2-\alpha} T u(t)\right)^{\prime}\right| \leq \frac{3 \alpha \Gamma(\alpha-1)}{2 \Gamma(2 \alpha-1)}\|u\|_{0},
\end{aligned}
$$

and so $\|T u\|_{1} \leq D\|u\|_{0} \leq D M$, where $D$ is as in (2.33). It means that $T \Omega$ is bounded.

Now, we show that the set of functions $B$ is equicontinuous on $(0.1]$, where $B=$ $\left\{t^{2-\alpha} \operatorname{Tu}(t): u \in \Omega\right\}$. 
Thai Advances in Difference Equations 2013, 2013:306

Page 10 of 24

http://www.advancesindifferenceequations.com/content/2013/1/306

In fact, for any $t_{1}, t_{2} \in(0,1]$ with $t_{1}<t_{2}$ and $u \in \Omega$, from (2.25), we have

$$
\begin{aligned}
& \left|t_{2}^{2-\alpha} \operatorname{Tu}\left(t_{2}\right)-t_{1}^{2-\alpha} T u\left(t_{1}\right)\right| \\
& \leq\left(t_{2}-t_{1}\right) \int_{0}^{1} G_{1}(s)|u(s)| d s \\
& \quad+\frac{1}{\Gamma(\alpha)}\left|t_{2}^{2-\alpha} \int_{0}^{t_{2}}\left(t_{2}-s\right)^{\alpha-1} u(s) d s-t_{1}^{2-\alpha} \int_{0}^{t_{1}}\left(t_{1}-s\right)^{\alpha-1} u(s) d s\right| \\
& \leq\left(t_{2}-t_{1}\right) \int_{0}^{1} G_{1}(s)|u(s)| d s+\frac{1}{\Gamma(\alpha)}\left|\int_{0}^{t_{1}}\left[t_{2}^{2-\alpha}\left(t_{2}-s\right)^{\alpha-1}-t_{1}^{2-\alpha}\left(t_{1}-s\right)^{\alpha-1}\right] u(s) d s\right| \\
& \quad+\frac{1}{\Gamma(\alpha)} t_{2}^{2-\alpha}\left|\int_{t_{1}}^{t_{2}}\left(t_{2}-s\right)^{\alpha-1} u(s) d s\right| .
\end{aligned}
$$

Again,

$$
\begin{aligned}
& \int_{0}^{1} G_{1}(s)|u(s)| d s=\int_{0}^{1} G_{1}(s) s^{\alpha-2}\left|s^{2-\alpha} u(s)\right| d s \leq M \int_{0}^{1} G_{1}(s) s^{2-\alpha} d s=\frac{M \alpha \Gamma(\alpha-1)}{2 \Gamma(2 \alpha-1)}, \\
& \left|\int_{0}^{t_{1}}\left[t_{2}^{2-\alpha}\left(t_{2}-s\right)^{\alpha-1}-t_{1}^{2-\alpha}\left(t_{1}-s\right)^{\alpha-1}\right] u(s) d s\right| \\
& \quad \leq\left(t_{2}^{2-\alpha}-t_{1}^{2-\alpha}\right) \int_{0}^{t_{1}}\left(t_{2}-s\right)^{\alpha-1}|u(s)| d s+t_{1}^{2-\alpha} \int_{0}^{t_{1}}\left[\left(t_{2}-s\right)^{\alpha-1}-\left(t_{1}-s\right)^{\alpha-1}\right]|u(s)| d s \\
& \leq M\left(t_{2}^{2-\alpha}-t_{1}^{2-\alpha}\right) \int_{0}^{t_{1}}\left(t_{2}-s\right)^{\alpha-1} s^{\alpha-2} d s \\
& \quad+M t_{1}^{2-\alpha} \int_{0}^{t_{1}}\left[\left(t_{2}-s\right)^{\alpha-1}-\left(t_{1}-s\right)^{\alpha-1}\right] s^{\alpha-2} d s,
\end{aligned}
$$

and

$$
\begin{aligned}
t_{2}^{2-\alpha}\left|\int_{t_{1}}^{t_{2}}\left(t_{2}-s\right)^{\alpha-1} u(s) d s\right| & \leq M \int_{t_{1}}^{t_{2}}\left(t_{2}-s\right)^{\alpha-1} s^{\alpha-2} d s \\
& \leq M \int_{t_{1}}^{t_{2}} s^{\alpha-2} d s=\frac{M}{\alpha-1}\left(t_{2}^{\alpha-1}-t_{1}^{\alpha-1}\right) .
\end{aligned}
$$

Thus, from (2.34), it follows that

$$
\begin{aligned}
& \left|t_{2}^{2-\alpha} \operatorname{Tu}\left(t_{2}\right)-t_{1}^{2-\alpha} T u\left(t_{1}\right)\right| \\
& \leq \frac{M \alpha \Gamma(\alpha-1)}{2 \Gamma(2 \alpha-1)}\left(t_{2}-t_{1}\right)+\frac{M\left(t_{2}^{2-\alpha}-t_{1}^{2-\alpha}\right)}{\Gamma(\alpha)} \int_{0}^{t_{1}}\left(t_{2}-s\right)^{\alpha-1} s^{\alpha-2} d s \\
& \quad+\frac{M t_{1}^{2-\alpha}}{\Gamma(\alpha)} \int_{0}^{t_{1}}\left[\left(t_{2}-s\right)^{\alpha-1}-\left(t_{1}-s\right)^{\alpha-1}\right] s^{\alpha-2} d s+\frac{M\left(t_{2}^{\alpha-1}-t_{1}^{\alpha-1}\right)}{(\alpha-1) \Gamma(\alpha)} .
\end{aligned}
$$

Again,

$$
\int_{0}^{t_{1}}\left(t_{2}-s\right)^{\alpha-1} s^{\alpha-2} d s \leq \int_{0}^{1} s^{\alpha-2} d s=\frac{1}{\alpha-1}
$$




$$
\begin{aligned}
t_{1}^{2-\alpha} & \int_{0}^{t_{1}}\left[\left(t_{2}-s\right)^{\alpha-1}-\left(t_{1}-s\right)^{\alpha-1}\right] s^{\alpha-2} d s \\
& =t_{1}^{2-\alpha}\left[\int_{0}^{t_{1}}\left(t_{2}-s\right)^{\alpha-1} s^{\alpha-2} d s-\int_{0}^{t_{1}}\left(t_{1}-s\right)^{\alpha-1} s^{\alpha-2} d s\right] \\
& =t_{1}^{2-\alpha}\left[t_{2}^{2(\alpha-1)} \int_{0}^{\frac{t_{1}}{t_{2}}}(1-\tau)^{\alpha-1} \tau^{\alpha-2} d \tau-t_{1}^{2(\alpha-1)} \int_{0}^{1}(1-\tau)^{\alpha-1} \tau^{\alpha-2} d \tau\right] \\
& \leq t_{1}^{2-\alpha}\left(t_{2}^{2(\alpha-1)}-t_{1}^{2(\alpha-1)}\right) \int_{0}^{1}(1-\tau)^{\alpha-1} \tau^{\alpha-2} d \tau \\
& =\left(t_{1}^{2-\alpha} \cdot t_{2}^{2(\alpha-1)}-t_{1}^{\alpha}\right) B(\alpha, \alpha-1) \\
& \leq\left(t_{2}^{\alpha}-t_{1}^{\alpha}\right) B(\alpha, \alpha-1),
\end{aligned}
$$

because

$$
\begin{aligned}
& \int_{0}^{t_{1}}\left(t_{2}-s\right)^{\alpha-1} s^{2-\alpha} d s \stackrel{s=t_{2} \tau}{=} t_{2}^{2(\alpha-1)} \int_{0}^{\frac{t_{1}}{t_{2}}}(1-\tau)^{\alpha-1} \tau^{\alpha-2} d \tau, \\
& \int_{0}^{t_{1}}\left(t_{1}-s\right)^{\alpha-1} s^{\alpha-2} d s \stackrel{s=t_{1} \tau}{=} t_{1}^{2(\alpha-1)} \int_{0}^{1}(1-\tau)^{\alpha-1} \tau^{\alpha-2} d \tau,
\end{aligned}
$$

and $t_{1}<t_{2}, 1<\alpha<2$.

Substituting (2.36)-(2.37) into (2.35), we have

$$
\begin{aligned}
\left|t_{2}^{2-\alpha} T u\left(t_{2}\right)-t_{1}^{2-\alpha} T u\left(t_{1}\right)\right| \leq & \frac{M \alpha \Gamma(\alpha-1)}{2 \Gamma(2 \alpha-1)}\left(t_{2}-t_{1}\right)+\frac{M\left(t_{2}^{2-\alpha}-t_{1}^{2-\alpha}\right)}{(\alpha-1) \Gamma(\alpha)} \\
& +\frac{M \Gamma(\alpha-1)}{\Gamma(2 \alpha-1)}\left(t_{2}^{\alpha}-t_{1}^{\alpha}\right)+\frac{M\left(t_{2}^{\alpha-1}-t_{1}^{\alpha-1}\right)}{(\alpha-1) \Gamma(\alpha)} .
\end{aligned}
$$

The above inequality shows that the set $B=\left\{t^{2-\alpha} T u(t) \mid u \in \Omega\right\}$ is equicontinuous on $(0,1]$.

Finally, we show that the set $B^{\prime}$ is also equicontinuous on $(0,1]$, where

$$
B^{\prime}=\left\{\left(t^{2-\alpha} T u(t)\right)^{\prime} \mid u \in \Omega\right\} .
$$

As before, for any $t_{1}, t_{2} \in(0,1]$ with $t_{1}<t_{2}$ and $u \in \Omega$, in view of (2.26), we have

$$
\begin{aligned}
&\left|\left(t_{2}^{2-\alpha} T u\left(t_{2}\right)\right)^{\prime}-\left(t_{1}^{2-\alpha} T u\left(t_{1}\right)\right)^{\prime}\right| \\
& \leq \frac{2-\alpha}{\Gamma(\alpha)}\left|t_{2}^{1-\alpha} \int_{0}^{t_{2}}\left(t_{2}-s\right)^{\alpha-1} u(s) d s-t_{1}^{1-\alpha} \int_{0}^{t_{1}}\left(t_{1}-s\right)^{\alpha-1} u(s) d s\right| \\
& \quad+\frac{1}{\Gamma(\alpha-1)}\left|t_{2}^{2-\alpha} \int_{0}^{t_{2}}\left(t_{2}-s\right)^{\alpha-2} u(s) d s-t_{1}^{2-\alpha} \int_{0}^{t_{1}}\left(t_{1}-s\right)^{\alpha-2} u(s) d s\right| \\
& \leq \frac{2-\alpha}{\Gamma(\alpha)}\left[\int_{0}^{t_{1}}\left|t_{2}^{1-\alpha}\left(t_{2}-s\right)^{\alpha-1}-t_{1}^{1-\alpha}\left(t_{1}-s\right)^{\alpha-1}\right||u(s)| d s\right. \\
&\left.+t_{2}^{1-\alpha} \int_{t_{1}}^{t_{2}}\left(t_{2}-s\right)^{\alpha-1}|u(s)| d s\right] \\
&+\frac{1}{\Gamma(\alpha-1)}\left[\int_{0}^{t_{1}}\left|t_{2}^{2-\alpha}\left(t_{2}-s\right)^{\alpha-2}-t_{1}^{2-\alpha}\left(t_{1}-s\right)^{\alpha-2}\right||u(s)| d s\right.
\end{aligned}
$$




$$
\begin{aligned}
& \left.+t_{2}^{2-\alpha} \int_{t_{1}}^{t_{2}}\left(t_{2}-s\right)^{\alpha-2}|u(s)| d s\right] \\
\leq & \frac{(2-\alpha) M}{\Gamma(\alpha)}\left[\int_{0}^{t_{1}}\left(\left(1-\frac{s}{t_{2}}\right)^{\alpha-1}-\left(1-\frac{s}{t_{1}}\right)^{\alpha-1}\right) s^{\alpha-2} d s+\int_{t_{1}}^{t_{2}}\left(1-\frac{s}{t_{2}}\right)^{\alpha-1} s^{\alpha-2} d s\right] \\
& +\frac{M}{\Gamma(\alpha-1)}\left[\int_{0}^{t_{1}}\left(\left(1-\frac{s}{t_{1}}\right)^{\alpha-2}-\left(1-\frac{s}{t_{2}}\right)^{\alpha-2}\right) s^{\alpha-2} d s\right. \\
& \left.+\int_{t_{1}}^{t_{2}}\left(1-\frac{s}{t_{2}}\right)^{\alpha-2} s^{\alpha-2} d s\right]
\end{aligned}
$$

noting that $|u(s)|=s^{\alpha-2} \cdot\left|s^{2-\alpha} u(s)\right| \leq s^{\alpha-2} M, s \in(0,1]$, and

$$
\left(1-\frac{s}{t_{2}}\right)^{\alpha-1}>\left(1-\frac{s}{t_{1}}\right)^{\alpha-1}, \quad\left(1-\frac{s}{t_{1}}\right)^{\alpha-2}>\left(1-\frac{s}{t_{2}}\right)^{\alpha-2}
$$

keeping in mind that $1<\alpha<2$.

For an arbitrary $\varepsilon>0$, take $\gamma=\frac{1}{2}\left(\frac{\Gamma(2 \alpha-2) \varepsilon}{3 M \Gamma(\alpha-1)}\right)^{\frac{1}{\alpha-1}}$. We can assume that $\varepsilon$ is small enough that $\gamma<1$. Since $t^{\alpha-1}$ is uniformly continuous on $[0,1]$, there exists $\delta_{1}>0$ such that

$$
0<t_{2}^{\alpha-1}-t_{1}^{\alpha-1}<\frac{(\alpha-1) \Gamma(\alpha) \varepsilon}{3(2-\alpha) M[(\alpha-1) B(\alpha, \alpha-1)+1]}
$$

when $0<t_{2}-t_{1}<\delta_{1}, t_{1}, t_{2} \in(0,1]$.

Moreover, because

$$
\begin{aligned}
& \int_{0}^{t_{1}}\left[\left(1-\frac{s}{t_{2}}\right)^{\alpha-1}-\left(1-\frac{s}{t_{1}}\right)^{\alpha-1}\right] s^{\alpha-2} d s \\
& \quad=\int_{0}^{t_{1}}\left(1-\frac{s}{t_{2}}\right)^{\alpha-1} s^{\alpha-2} d s-\int_{0}^{t_{1}}\left(1-\frac{s}{t_{1}}\right)^{\alpha-1} s^{\alpha-2} d s \\
& \quad=t_{2}^{\alpha-1} \int_{0}^{\frac{t_{1}}{t_{2}}}(1-\tau)^{\alpha-1} \tau^{\alpha-2} d \tau-t_{1}^{\alpha-1} \int_{0}^{1}(1-\tau)^{\alpha-1} \tau^{\alpha-2} d \tau \\
& \quad \leq\left(t_{2}^{\alpha-1}-t_{1}^{\alpha-1}\right) \int_{0}^{1}(1-\tau)^{\alpha-1} \tau^{\alpha-2} d \tau=\left(t_{2}^{\alpha-1}-t_{1}^{\alpha-1}\right) B(\alpha, \alpha-1)
\end{aligned}
$$

and

$$
\int_{t_{1}}^{t_{2}}\left(1-\frac{s}{t_{2}}\right)^{\alpha-1} s^{\alpha-2} d s<\int_{t_{1}}^{t_{2}} s^{\alpha-2} d s=\frac{1}{\alpha-1}\left(t_{2}^{\alpha-1}-t_{1}^{\alpha-1}\right)
$$

we obtain

$$
\begin{aligned}
& \frac{(2-\alpha) M}{\Gamma(\alpha)}\left[\int_{0}^{t_{1}}\left(\left(1-\frac{s}{t_{2}}\right)^{\alpha-1}-\left(1-\frac{s}{t_{1}}\right)^{\alpha-1}\right) s^{\alpha-2} d s+\int_{t_{1}}^{t_{2}}\left(1-\frac{s}{t_{2}}\right)^{\alpha-1} s^{\alpha-2} d s\right] \\
& \leq \frac{(2-\alpha) M}{\Gamma(\alpha)}\left[B(\alpha, \alpha-1)+\frac{1}{\alpha-1}\right]\left(t_{2}^{\alpha-1}-t_{1}^{\alpha-1}\right)<\frac{\varepsilon}{3}
\end{aligned}
$$

when $0<t_{2}-t_{1}<\delta_{1}, t_{1}, t_{2} \in(0,1]$. 
(1) If $0<t_{1}<\gamma$ and $t_{1}<t_{2}$ with $t_{2}-t_{1}<\gamma$, then

$$
\begin{aligned}
& \frac{M}{\Gamma(\alpha-1)} \int_{0}^{t_{1}}\left[\left(1-\frac{s}{t_{1}}\right)^{\alpha-2}-\left(1-\frac{s}{t_{2}}\right)^{\alpha-2}\right] s^{\alpha-2} d s \\
& <\frac{M}{\Gamma(\alpha-1)} \int_{0}^{t_{1}}\left(1-\frac{s}{t_{1}}\right)^{\alpha-2} s^{\alpha-2} d s \\
& \stackrel{s=t_{1} \tau}{=} \frac{M t_{1}^{\alpha-1}}{\Gamma(\alpha-1)} \int_{0}^{1}(1-\tau)^{\alpha-2} \tau^{\alpha-2} d \tau \\
& \leq \frac{M \Gamma(\alpha-1)}{\Gamma(2 \alpha-2)} \gamma^{\alpha-1}<\frac{\varepsilon}{3}
\end{aligned}
$$

and

$$
\begin{aligned}
& \frac{M}{\Gamma(\alpha-1)} \int_{t_{1}}^{t_{2}}\left(1-\frac{s}{t_{2}}\right)^{\alpha-2} s^{\alpha-2} d s \stackrel{s=t_{2} \tau}{=} \frac{M t_{2}^{\alpha-1}}{\Gamma(\alpha-1)} \int_{\frac{t_{1}}{t_{2}}}^{1}(1-\tau)^{\alpha-2} \tau^{\alpha-2} d \tau \\
&<\frac{M t_{2}^{\alpha-1}}{\Gamma(\alpha-1)} \int_{0}^{1}(1-\tau)^{\alpha-2} \tau^{\alpha-2} d \tau \\
&<\frac{2^{\alpha-1} M \Gamma(\alpha-1)}{\Gamma(2 \alpha-2)} \gamma^{\alpha-1}=\frac{\varepsilon}{3}
\end{aligned}
$$

because $0<t_{2}<2 \gamma$.

Consequently, by (2.40)-(2.41) we have

$$
\begin{aligned}
& \frac{M}{\Gamma(\alpha-1)}\left[\int_{0}^{t_{1}}\left(\left(1-\frac{s}{t_{1}}\right)^{\alpha-2}-\left(1-\frac{s}{t_{2}}\right)^{\alpha-2}\right) s^{\alpha-2} d s+\int_{t_{1}}^{t_{2}}\left(1-\frac{s}{t_{2}}\right)^{\alpha-2} s^{\alpha-2} d s\right] \\
& \quad<\frac{2 \varepsilon}{3}
\end{aligned}
$$

when $0<t_{1}<\gamma$ with $0<t_{2}-t_{1}<\gamma$.

(2) If $\gamma \leq t_{1}<t_{2}$, then

$$
\begin{aligned}
& \int_{0}^{t_{1}}\left[\left(1-\frac{s}{t_{1}}\right)^{\alpha-2}-\left(1-\frac{s}{t_{2}}\right)^{\alpha-2}\right] s^{\alpha-2} d s \\
& =t_{1}^{\alpha-1} \int_{0}^{1}(1-\tau)^{\alpha-2} \tau^{\alpha-2} d \tau-t_{2}^{\alpha-1} \int_{0}^{\frac{t_{1}}{t_{2}}}(1-\tau)^{\alpha-2} \tau^{\alpha-2} d \tau \\
& =\left(t_{1}^{\alpha-1}-t_{2}^{\alpha-1}\right) \int_{0}^{1}(1-\tau)^{\alpha-2} \tau^{\alpha-2} d \tau+t_{2}^{\alpha-1} \int_{\frac{t_{1}}{t_{2}}}^{1}(1-\tau)^{\alpha-2} \tau^{\alpha-2} d \tau \\
& \quad<t_{2}^{\alpha-1} \int_{\frac{t_{1}}{t_{2}}}^{1}(1-\tau)^{\alpha-2} \tau^{\alpha-2} d \tau<\int_{\frac{t_{1}}{t_{2}}}^{1}(1-\tau)^{\alpha-2} \tau^{\alpha-2} d \tau,
\end{aligned}
$$

and

$$
\int_{t_{1}}^{t_{2}}\left(1-\frac{s}{t_{2}}\right)^{\alpha-2} s^{\alpha-2} d s=t_{2}^{\alpha-1} \int_{\frac{t_{1}}{t_{2}}}^{1}(1-\tau)^{\alpha-2} \tau^{\alpha-2} d \tau \leq \int_{\frac{t_{1}}{t_{2}}}^{1}(1-\tau)^{\alpha-2} \tau^{\alpha-2} .
$$


Hence, (2.43)-(2.44) imply that

$$
\begin{aligned}
& \frac{M}{\Gamma(\alpha-1)}\left[\int_{0}^{t_{1}}\left(\left(1-\frac{s}{t_{2}}\right)^{\alpha-2}-\left(1-\frac{s}{t_{1}}\right)^{\alpha-2}\right) s^{\alpha-2} d s+\int_{t_{1}}^{t_{2}}\left(1-\frac{s}{t_{2}}\right)^{\alpha-1} s^{\alpha-2} d s\right] \\
& <\frac{2 M}{\Gamma(\alpha-1)} \int_{\frac{t_{1}}{t_{2}}}^{1}(1-\tau)^{\alpha-2} \tau^{\alpha-2} d \tau .
\end{aligned}
$$

Because $\int_{0}^{1}(1-\tau)^{\alpha-2} \tau^{\alpha-2} d \tau=B(\alpha-1, \alpha-1)<\infty$, there exists $\sigma \in(0,1)$ such that

$$
\int_{\eta}^{1}(1-\tau)^{\alpha-2} \tau^{\alpha-2} d \tau<\frac{\Gamma(\alpha-1)}{3 M} \varepsilon
$$

when $0<1-\eta<\sigma$.

Take $0<\delta_{2} \leq \gamma \sigma$, then from (2.46), it follows that

$$
\int_{\frac{t_{1}}{t_{2}}}^{1}(1-\tau)^{\alpha-2} \tau^{\alpha-2} d \tau<\frac{\Gamma(\alpha-1)}{3 M} \varepsilon
$$

when $0<t_{2}-t_{1}<\delta_{2}$, noting that $0<1-\frac{t_{1}}{t_{2}}=\frac{t_{2}-t_{1}}{t_{2}}<\frac{\delta_{2}}{\gamma} \leq \sigma$ because $t_{2}>t_{1} \geq \gamma$.

So, from (2.45) together with (2.47), it follows that

$$
\begin{aligned}
& \frac{M}{\Gamma(\alpha-1)}\left[\int_{0}^{t_{1}}\left(\left(1-\frac{s}{t_{2}}\right)^{\alpha-2}-\left(1-\frac{s}{t_{1}}\right)^{\alpha-2}\right) s^{\alpha-2} d s+\int_{t_{1}}^{t_{2}}\left(1-\frac{s}{t_{2}}\right)^{\alpha-1} s^{\alpha-2} d s\right] \\
& \quad<\frac{2 \varepsilon}{3}
\end{aligned}
$$

when $\gamma \leq t_{1}$ and $0<t_{2}-t_{1}<\delta_{2}$.

Now, take $\delta=\min \left\{\delta_{1}, \delta_{2}, \gamma\right\}$. Then, when $0<t_{2}-t_{1}<\delta$, by (2.38), (2.39), (2.42) and (2.48), we have

$$
\left|\left(t_{2}^{2-\alpha} T u\left(t_{2}\right)\right)^{\prime}-\left(t_{1}^{2-\alpha} T u\left(t_{1}\right)\right)^{\prime}\right|<\varepsilon
$$

The above inequality (2.49) shows that the set $B^{\prime}$ is equicontinuous on $(0,1]$. As a consequence of the Arzela-Ascoli theorem, we have that $T \Omega$ is a compact set in $X_{1}$. The proof is complete.

Finally, for the remainder of this section, we give the following lemma, which will be used to obtain our main results.

Lemma 2.6 ([35]) Let E be a Banach space. Assume that $\Omega$ is an open bounded subset of $E$ with $\theta \in \Omega$, and let $A: \bar{\Omega} \rightarrow E$ be a completely continuous operator such that

$$
\|A u\| \leq\|u\|, \quad \forall u \in \partial \Omega .
$$

Then $A$ has a fixed point in $\Omega$. 


\section{Main results}

Let us introduce some assumptions which will be used throughout this paper.

$\left(\mathrm{H}_{1}\right) f \in C((0,1] \times \mathbb{R} \times \mathbb{R}, \mathbb{R})$.

$\left(\mathrm{H}_{2}\right) \sup _{t \in(0,1]}\left|t^{2-\alpha} f(t, 0,0)\right|<\infty$.

$\left(\mathrm{H}_{3}\right)$ There exist two constants $L_{1}>0, L_{2}>0$ such that

$$
\left|f\left(t, x_{2}, y_{2}\right)-f\left(t, x_{1}, y_{1}\right)\right| \leq L_{1}\left|x_{2}-x_{1}\right|+L_{2} t\left|y_{2}-y_{1}\right|
$$

for all $x_{i}, y_{i} \in \mathbb{R}, i=1,2$, and $t \in(0,1]$.

$\left(\mathrm{H}_{4}\right)$ There exist a function $\phi \in X_{0}$ and constants $N_{1}>0, N_{2}>0, \theta_{1}, \theta_{2} \in(0,1)$ such that

$$
|f(t, x, y)| \leq \phi(t)+N_{1}|x|^{\theta_{1}}+N_{2} t|y|^{\theta_{2}}
$$

for all $x_{i}, y_{i} \in \mathbb{R}, i=1,2$, and $t \in(0,1]$.

We define two operators $F, T$ on $X_{1}$ as follows:

$$
A u=T F u, \quad F u=f\left(t, u, u^{\prime}\right), \quad t \in(0,1], \text { for } u \in X_{1},
$$

where $T$ is defined as before.

We first establish the following lemma to obtain our results.

Lemma 3.1 Suppose that $\left(\mathrm{H}_{1}\right),\left(\mathrm{H}_{2}\right),\left(\mathrm{H}_{3}\right)$ hold. Then the operator $A$ maps $X_{1}$ into $X_{1}$.

Proof For any $u \in X_{1}$, by $\left(\mathrm{H}_{3}\right)$, we have

$$
\left|f\left(t, u, u^{\prime}\right)-f(t, 0,0)\right| \leq L_{1}|u(t)|+L_{2} t\left|u^{\prime}(t)\right|, \quad t \in(0,1] .
$$

So,

$$
\left|f\left(t, u(t), u^{\prime}(t)\right)\right| \leq|f(t, 0,0)|+L_{1}|u(t)|+L_{2} t\left|u^{\prime}(t)\right|, \quad t \in(0,1] .
$$

Again,

$$
|u(t)|=\left|t^{\alpha-2} t^{2-\alpha} u(t)\right| \leq t^{\alpha-2}\|u\|_{1}, \quad t \in(0,1],
$$

and so

$$
\begin{aligned}
\left|u^{\prime}(t)\right| & =\left|t^{\alpha-2}\left(t^{2-\alpha} u(t)\right)^{\prime}+(\alpha-2) t^{-1} u(t)\right| \\
& \leq t^{\alpha-2}\left|\left(t^{2-\alpha} u(t)\right)^{\prime}\right|+(2-\alpha) t^{-1}|u(t)| \\
& \leq t^{\alpha-2}\|u\|_{1}+(2-\alpha) t^{\alpha-3}\|u\|_{1} \\
& <t^{\alpha-3}(3-\alpha)\|u\|_{1}, \quad t \in(0,1] .
\end{aligned}
$$

Hence, by (3.1)-(3.3), we have

$$
\left|f\left(t, u(t), u^{\prime}(t)\right)\right| \leq|f(t, 0,0)|+\left(L_{1}+(3-\alpha) L_{2}\right) t^{\alpha-2}\|u\|_{1}, \quad t \in(0,1],
$$


and so

$$
\left|t^{2-\alpha} f\left(t, u(t), u^{\prime}(t)\right)\right| \leq\left|t^{2-\alpha} f(t, 0,0)\right|+\left(L_{1}+(3-\alpha) L_{2}\right)\|u\|_{1}, \quad t \in(0,1] .
$$

Again, from $\left(\mathrm{H}_{2}\right)$ and by letting $K=\sup _{t \in(0,1]}\left|t^{2-\alpha} f(t, 0,0)\right|$, we have

$$
|f(t, 0,0)|=t^{\alpha-2}\left|t^{2-\alpha} f(t, 0,0)\right| \leq K t^{\alpha-2}, \quad t \in(0,1],
$$

and so $f(t, 0,0) \in L(0,1)$. Thus, according to hypothesis $\left(\mathrm{H}_{1}\right)$ and (3.4)-(3.5), we know that $f\left(t, u, u^{\prime}\right) \in C(0,1] \cap L(0,1)$ and $\sup _{t \in(0,1]}\left|t^{2-\alpha} f\left(t, u, u^{\prime}\right)\right|<\infty$. That is, Fu $\in X_{0}$. Therefore, in view of Lemma 2.5, it follows that $A u \in X_{1}$. Thus, $A: X_{1} \rightarrow X_{1}$. The proof is complete.

The following lemma is significant to obtaining the result in this article.

Lemma 3.2 Suppose that $\left(\mathrm{H}_{1}\right)$ and $\left(\mathrm{H}_{4}\right)$ hold. Then the operator $A: X_{1} \rightarrow X_{1}$ is completely continuous.

Proof We first show that the operator $F$ maps $X_{1}$ into $X_{0}$ under $\left(\mathrm{H}_{4}\right)$.

In fact, for any $u \in X_{1}$, by an argument similar to (3.4)-(3.5), from $\left(\mathrm{H}_{4}\right)$ combined with (3.2)-(3.3), it follows that

$$
\begin{aligned}
\left|f\left(t, u(t), u^{\prime}(t)\right)\right| & \leq \phi(t)+N_{1}|u(t)|^{\theta_{1}}+N_{2} t\left|u^{\prime}(t)\right|^{\theta_{2}} \\
& \leq \phi(t)+N_{1} t^{(\alpha-2) \theta_{1}}\|u\|_{1}^{\theta_{1}}+N_{2} t(3-\alpha)^{\theta_{2}} t^{(\alpha-3) \theta_{2}}\|u\|_{1}^{\theta_{2}} \\
& \leq \phi(t)+t^{(\alpha-2)}\left(N_{1}\|u\|_{1}^{\theta_{1}}+N_{2}(3-\alpha)\|u\|_{1}^{\theta_{2}}\right), \quad t \in(0,1],
\end{aligned}
$$

and so

$$
\left|t^{2-\alpha} f\left(t, u(t), u^{\prime}(t)\right)\right| \leq t^{2-\alpha} \phi(t)+N_{1}\|u\|_{1}^{\theta_{1}}+N_{2}(3-\alpha)\|u\|_{1}^{\theta_{2}}, \quad t \in(0,1]
$$

noting that $\theta_{1}, \theta_{2} \in(0,1)$ and $1<\alpha<2$.

Thus, according to hypothesis $\left(\mathrm{H}_{1}\right)$, formulae (3.6)-(3.7) ensure that $F u \in X_{0}$, noting that $\phi \in X_{0}$.

Now, we prove that the operator $A: X_{1} \rightarrow X_{1}$ is completely continuous.

First of all, in view of Lemma 2.5, we know that the operator $A: X_{1} \rightarrow X_{1}$ because of the fact that $F: X_{1} \rightarrow X_{0}$ and $A=T F$.

It remains to show that the operator $A$ is completely continuous. The following proof is divided into two steps.

Step 1. We show that the operator $A$ is compact on $X_{1}$.

In fact, assuming that $\Omega$ is an arbitrary set in $X_{1}$, there exists $M>0$ such that $\|u\|_{1} \leq M$ for all $u \in \Omega$. Thus, from (3.7), it follows that

$$
\|F u\|_{0} \leq \sup _{t \in(0,1]} t^{2-\alpha} \phi(t)+N_{1} M^{\theta_{1}}+N_{2}(3-\alpha) M^{\theta_{2}} \stackrel{\Delta}{=} N .
$$

Therefore, the set $B=\{h \mid h=F u, u \in \Omega\}$ is bounded in $X_{0}$, and therefore, $T B$ is a compact set in view of Lemma 2.5. That is, $A \Omega$ is a compact set, owing to the fact that $A=T F$ and $B=F \Omega$. Hence, $A$ is a compact operator. 
Step 2. We show that the operator $A$ is continuous on $X_{1}$.

Let $\left\{u_{n}\right\}_{n=1}^{\infty}$ be an arbitrary sequence in $X_{1}$ with $u_{n} \rightarrow u_{0} \in X_{1}$. Then there exists $M>0$ such that $\left\|u_{n}\right\|_{1} \leq M, n=0,1,2, \ldots$. Thus, from (3.7), it follows that

$$
\begin{aligned}
\left|t^{2-\alpha} F u_{n}(t)\right| & \leq\left|t^{2-\alpha} \phi(t)\right|+N_{1}\left\|u_{n}\right\|_{1}^{\theta_{1}}+N_{2}(3-\alpha)\left\|u_{n}\right\|_{1}^{\theta_{2}} \\
& \leq \sup _{t \in(0,1]}\left|t^{2-\alpha} \phi(t)\right|+N_{1} M^{\theta_{1}}+N_{2}(3-\alpha) M^{\theta_{2}} \stackrel{\Delta}{=} N
\end{aligned}
$$

for $n=0,1,2, \ldots$.

The following proof is divided into two parts.

Part 1. By (2.25), we have

$$
\begin{aligned}
\mid t^{2-\alpha} & \left(A u_{n}(t)-A u_{0}(t)\right) \mid \\
\leq & t \int_{0}^{1} G_{1}(s)\left|F u_{n}(s)-F u_{0}(s)\right| d s \\
\quad & +\int_{0}^{1}\left|G_{2}(s)\right| \cdot\left|F u_{n}(s)-F u_{0}(s)\right| d s+\frac{1}{\Gamma(\alpha)} t^{2-\alpha} \int_{0}^{t}(t-s)^{\alpha-1}\left|F u_{n}(s)-F u_{0}(s)\right| d s \\
\leq & \int_{0}^{1}\left(G_{1}(s)+\left|G_{2}(s)\right|+\frac{1}{\Gamma(\alpha)}(1-s)^{\alpha-1}\right)\left|F u_{n}(s)-u_{0}(s)\right| d s, \quad t \in(0,1] .
\end{aligned}
$$

By (2.28)-(2.29), we get

$$
\int_{0}^{1}\left(G_{1}(s)+\left|G_{2}(s)\right|+\frac{1}{\Gamma(\alpha)}(1-s)^{\alpha-1}\right) s^{\alpha-2} d s \leq \frac{5 \alpha+2}{4 \Gamma(2 \alpha-1)}<+\infty .
$$

So, for any $\varepsilon>0$, by the absolute continuity of Lebesgue integral, there exists $0<\delta<1$ such that

$$
2 N \int_{0}^{\delta}\left(G_{1}(s)+\left|G_{2}(s)\right|+\frac{1}{\Gamma(\alpha)}(1-s)^{\alpha-1}\right) s^{\alpha-2} d s<\frac{\varepsilon}{2},
$$

and therefore,

$$
\int_{0}^{\delta}\left(G_{1}(s)+\left|G_{2}(s)\right|+\frac{1}{\Gamma(\alpha)}(1-s)^{\alpha-1}\right)\left|F u_{n}(s)-F u_{0}(s)\right| d s<\frac{\varepsilon}{2}
$$

holds for $n=1,2, \ldots$, because $\left|F u_{n}(s)-F u_{0}(s)\right| \leq 2 N s^{\alpha-2}, s \in(0, \delta], n=1,2, \ldots$, observing (3.8).

On the other hand, for all $t \in[\delta, 1]$, formulae (3.2)-(3.3) imply that

$$
\begin{aligned}
& \left|u_{n}(t)\right| \leq t^{\alpha-2}\left\|u_{n}\right\|_{1} \leq \delta^{\alpha-2} M, \quad n=0,1,2, \ldots, \\
& \left|u_{n}^{\prime}(t)\right| \leq(3-\alpha) t^{\alpha-3}\left\|u_{n}\right\|_{1} \leq 2 \delta^{\alpha-3} M, \quad n=0,1,2, \ldots
\end{aligned}
$$

and

$$
\begin{aligned}
& \left|u_{n}(s)-u_{0}(s)\right| \leq \delta^{\alpha-2}\left\|u_{n}-u_{0}\right\|_{1}, \quad n \geq 1, \\
& \left|\left(u_{n}(t)-u_{0}(t)\right)^{\prime}\right| \leq 2 \delta^{\alpha-3}\left\|u_{n}-u_{0}\right\|_{1}, \quad n \geq 1 .
\end{aligned}
$$


Since $f(t, x, y)$ is uniformly continuous on $[\delta, 1] \times\left[-\delta^{\alpha-2} M, \delta^{\alpha-2} M\right] \times\left[-2 \delta^{\alpha-3} M, 2 \delta^{\alpha-3} M\right]$, from $\left(\mathrm{H}_{1}\right)$, for $\varepsilon$ given as before, there exists $K_{1} \geq 1$ such that

$$
\left|F u_{n}(s)-F u_{0}(s)\right|<\frac{d}{2} \varepsilon, \quad s \in[\delta, 1]
$$

when $n \geq K_{1}$, noting that (3.11)-(3.12), where $d=\left(\int_{\delta}^{1}\left(G_{1}(s)+\left|G_{2}(s)\right|+\frac{(1-s)^{\alpha-1}}{\Gamma(\alpha)}\right) d s\right)^{-1}$.

Thus, when $n \geq K_{1}$, formula (3.13) implies that

$$
\int_{\delta}^{1}\left(G_{1}(s)+\left|G_{2}(s)\right|+\frac{1}{\Gamma(\alpha)}(1-s)^{\alpha-1}\right)\left|F u_{n}(s)-F u_{0}(s)\right| d s<\frac{\varepsilon}{2}
$$

holds for $n=1,2, \ldots$.

Substituting (3.10) and (3.14) into (3.9), we have that

$$
\left|t^{2-\alpha}\left(A u_{n}(t)-A u(t)\right)\right|<\varepsilon, \quad t \in(0,1]
$$

when $n \geq K_{1}$, and so

$$
\sup _{t \in(0,1]}\left|t^{2-\alpha}\left(A u_{n}(t)-A u(t)\right)\right| \leq \varepsilon
$$

when $n \geq K_{1}$.

Part 2. By (2.26), we have

$$
\begin{aligned}
\left|\left(t^{2-\alpha}\left(A u_{n}(t)-A u_{0}(t)\right)\right)^{\prime}\right| & \\
\leq & \int_{0}^{1} G_{1}(s)\left|F u_{n}(s)-F u_{0}(s)\right| d s \\
& +\frac{2-\alpha}{\Gamma(\alpha)} t^{1-\alpha} \int_{0}^{t}(t-s)^{\alpha-1}\left|F u_{n}(s)-F u_{0}(s)\right| d s \\
& +\frac{t^{2-\alpha}}{\Gamma(\alpha-1)} \int_{0}^{t}(t-s)^{\alpha-2}\left|F u_{n}(s)-F u_{0}(s)\right| d s \\
= & \int_{0}^{1} G_{1}(s)\left|F u_{n}(s)-F u_{0}(s)\right| d s+\frac{2-\alpha}{\Gamma(\alpha)} \int_{0}^{t}\left(1-\frac{s}{t}\right)^{\alpha-1}\left|F u_{n}(s)-F u_{0}(s)\right| d s \\
& +\frac{1}{\Gamma(\alpha-1)} \int_{0}^{t}\left(1-\frac{s}{t}\right)^{\alpha-2}\left|F u_{n}(s)-F u_{0}(s)\right| d s \\
< & \int_{0}^{1} G(s)\left|F u_{n}(s)-F u_{0}(s)\right| d s+\frac{1}{\Gamma(\alpha)} \int_{0}^{t}\left(1-\frac{s}{t}\right)^{\alpha-2}\left|F u_{n}(s)-F u_{0}(s)\right| d s
\end{aligned}
$$

because of the fact that $\frac{2-\alpha}{\Gamma(\alpha)}+\frac{1}{\Gamma(\alpha-1)}=\frac{1}{\Gamma(\alpha)}$ and $\left(1-\frac{s}{t}\right)^{\alpha-1}<\left(1-\frac{s}{t}\right)^{\alpha-2}$.

For $\varepsilon$ given as before, take $0<\delta_{1}<\min \left\{1,\left(\frac{\Gamma(\alpha) \varepsilon}{6 N \cdot B(\alpha-1, \alpha-1)}\right) \frac{1}{\alpha-1}\right\}$, where $N$ is given by (3.8).

(i) If $t \in\left(0, \delta_{1}\right]$, then from (3.8) it follows that

$$
\begin{aligned}
& \frac{1}{\Gamma(\alpha)} \int_{0}^{t}\left(1-\frac{s}{t}\right)^{\alpha-2}\left|F u_{n}(s)-F u_{0}(s)\right| d s \\
& \quad=\frac{1}{\Gamma(\alpha)} \int_{0}^{t}\left(1-\frac{s}{t}\right)^{\alpha-2} s^{\alpha-2}\left|s^{2-\alpha}\left(F u_{n}(s)-F u_{0}(s)\right)\right| d s
\end{aligned}
$$




$$
\begin{aligned}
& \leq \frac{2 N}{\Gamma(\alpha)} \int_{0}^{t}\left(1-\frac{s}{t}\right)^{\alpha-2} s^{\alpha-2} d s \\
& \stackrel{s=t \tau}{=} \frac{2 N}{\Gamma(\alpha)} t^{\alpha-1} \int_{0}^{1}(1-\tau)^{\alpha-2} \tau^{\alpha-2} d \tau \\
& \leq \frac{2 N}{\Gamma(\alpha)} \delta_{1}^{\alpha-1} B(\alpha-1, \alpha-1)<\frac{\varepsilon}{3} .
\end{aligned}
$$

(ii) If $t \in\left(\delta_{1}, 1\right]$, then

$$
\begin{aligned}
& \frac{1}{\Gamma(\alpha)} \int_{0}^{t}\left(1-\frac{s}{t}\right)^{\alpha-2}\left|F u_{n}(s)-F u_{0}(s)\right| d s \\
& =\frac{1}{\Gamma(\alpha)} \int_{0}^{\delta_{1}}\left(1-\frac{s}{t}\right)^{\alpha-2}\left|F u_{n}(s)-F u_{0}(s)\right| d s \\
& \quad+\frac{1}{\Gamma(\alpha)} \int_{\delta_{1}}^{t}\left(1-\frac{s}{t}\right)^{\alpha-2}\left|F u_{n}(s)-F u_{0}(s)\right| d s
\end{aligned}
$$

Again,

$$
\begin{aligned}
& \frac{1}{\Gamma(\alpha)} \int_{0}^{\delta_{1}}\left(1-\frac{s}{t}\right)^{\alpha-2}\left|F u_{n}(s)-F u_{0}(s)\right| d s \\
& \leq \frac{1}{\Gamma(\alpha)} \int_{0}^{\delta_{1}}\left(1-\frac{s}{\delta_{1}}\right)^{\alpha-2}\left|F u_{n}(s)-F u_{0}(s)\right| d s \\
& \quad \leq \frac{2 N}{\Gamma(\alpha)} \int_{0}^{\delta_{1}}\left(1-\frac{s}{\delta_{1}}\right)^{\alpha-2} s^{\alpha-2} d s \\
& \stackrel{s=\delta_{1} \tau}{=} \frac{2 N}{\Gamma(\alpha)} \delta_{1}^{\alpha-1} \int_{0}^{1}(1-\tau)^{\alpha-2} \tau^{\alpha-2} d s \\
& =\frac{2 N}{\Gamma(\alpha)} \delta_{1}^{\alpha-1} B(\alpha-1, \alpha-1)<\frac{\varepsilon}{3} .
\end{aligned}
$$

By an argument similar to (3.13), we can know that there exists $K_{2} \geq 1$ such that

$$
\left|F u_{n}(s)-F u_{0}(s)\right|<\frac{d_{1}}{3} \varepsilon, \quad s \in\left[\delta_{1}, 1\right]
$$

when $n \geq K_{2}$, where $d_{1}=(\alpha-1) \Gamma(\alpha)$.

Thus, from (3.20), for $t \in\left(\delta_{1}, 1\right]$, it follows that

$$
\begin{aligned}
\frac{1}{\Gamma(\alpha)} \int_{\delta_{1}}^{t}\left(1-\frac{s}{t}\right)^{\alpha-2}\left|F u_{n}(s)-F u_{0}(s)\right| d s & <\frac{d_{1} \varepsilon}{3 \Gamma(\alpha)} \int_{\delta_{1}}^{t}\left(1-\frac{s}{t}\right)^{\alpha-2} d s \\
& <\frac{d_{1} \varepsilon}{3(\alpha-1) \Gamma(\alpha)}=\frac{\varepsilon}{3}
\end{aligned}
$$

when $n \geq K_{2}$.

By (3.18) together with (3.19) and (3.21), we have

$$
\frac{1}{\Gamma(\alpha)} \int_{0}^{t}\left(1-\frac{s}{t}\right)^{\alpha-2}\left|F u_{n}(s)-F u_{0}(s)\right| d s<\frac{2 \varepsilon}{3}, \quad t \in(0,1]
$$

when $n \geq K_{2}$. 
On the other hand, from the proof in Part 1 (see the deducing on (3.10) and (3.14)), there exists $K_{3}$ such that the inequality

$$
\int_{0}^{1} G_{1}(s)\left|F u_{n}(s)-F u_{0}(s)\right| d s<\frac{\varepsilon}{3}
$$

holds when $n \geq K_{3}$.

Define $L=\max \left\{K_{2}, K_{3}\right\}$. From (3.16) together with (3.17), (3.22) and (3.23), we have

$$
\left|\left(t^{2-\alpha}\left(A u_{n}(t)-A u_{0}(t)\right)\right)^{\prime}\right|<\varepsilon, \quad t \in(0,1]
$$

when $n \geq L$, and so

$$
\sup _{t \in(0,1]}\left|\left(t^{2-\alpha}\left(A u_{n}(t)-A u_{0}(t)\right)\right)^{\prime}\right| \leq \varepsilon
$$

when $n \geq L$.

Formulae (3.15), (3.24) yield that $A u_{n} \rightarrow A u_{0}$ in $X_{1}$. That is, the operator $A$ is continuous on $X_{1}$. The proof is complete.

In view of Lemma 2.3, it is easy to know that $u \in X_{1}$ is a solution of BVP (1.1)-(1.2) if and only if $u \in X_{1}$ is a fixed point of the operator $A$. Therefore, we can focus on seeking the existence of a fixed point of $A$ in $X_{1}$.

Let $D=\frac{(5 \alpha+2) \Gamma(\alpha-1)}{4 \Gamma(2 \alpha-1)}\left(L_{1}+(3-\alpha) L_{2}\right)$, where $L_{1}, L_{2}$ is given by $\left(\mathrm{H}_{3}\right)$.

We are now in a position to state the first theorem in the present paper.

Theorem 3.1 Suppose that $\left(\mathrm{H}_{1}\right)-\left(\mathrm{H}_{3}\right)$ hold. If $D<1$, then BVP (1.1)-(1.2) has a unique solution.

Proof According to $\left(\mathrm{H}_{1}\right)-\left(\mathrm{H}_{2}\right)$, we know that $A: X_{1} \rightarrow X_{1}$ by Lemma 3.1. By an argument similar to (2.30), for any $u, v \in X_{1}$, in view of $\left(\mathrm{H}_{3}\right)$ together with (2.25), (2.28) and (2.29), we have

$$
\begin{aligned}
\mid t^{2-\alpha} & (A u(t)-A v(t)) \mid \\
\leq & t \int_{0}^{1} G_{1}(s)|F u(s)-F v(s)| d s \\
& +\int_{0}^{1}\left|G_{2}(s)\right| \cdot|F u(s)-F v(s)| d s+\frac{t^{2-\alpha}}{\Gamma(\alpha)} \int_{0}^{t}(t-s)^{\alpha-1}|F u(s)-F v(s)| d s, \\
\leq & {\left[t \int_{0}^{1} G_{1}(s) s^{\alpha-2} d s+\int_{0}^{1}\left|G_{2}(s)\right| s^{\alpha-2} d s+\frac{t^{2-\alpha}}{\Gamma(\alpha)} \int_{0}^{t}(t-s)^{\alpha-1} s^{\alpha-2} d s\right] } \\
& \cdot\left(L_{1}+(3-\alpha) L_{2}\right)\|u-v\|_{1} \\
\leq & \frac{(5 \alpha+2) \Gamma(\alpha-1)\left(L_{1}+(3-\alpha) L_{2}\right)}{4 \Gamma(2 \alpha-1)}\|u-v\|_{1} \\
\doteq & D_{1}\|u-v\|_{1}, \quad t \in(0,1],
\end{aligned}
$$


because

$$
\begin{aligned}
|F u(s)-F v(s)| & \leq L_{1}|u(s)-v(s)|+L_{2} s\left|(u(s)-v(s))^{\prime}\right| \\
& \leq s^{\alpha-2}\left(L_{1}+(3-\alpha) L_{2}\right)\|u-v\|_{1}, \quad s \in(0,1]
\end{aligned}
$$

by (3.2)-(3.3).

Thus,

$$
\sup _{t \in(0,1]}\left|t^{2-\alpha}(A u(t)-A v(t))\right| \leq D_{1}\|u-v\|_{1} .
$$

Similarly, from (2.26) together with (2.28), (2.31), we have

$$
\sup _{t \in(0,1]}\left|\left(t^{2-\alpha}(A u(t)-A v(t))\right)^{\prime}\right| \leq D_{2}\|u-v\|_{1}
$$

where $D_{2}=\frac{3 \alpha(\Gamma \alpha-1)}{2 \Gamma(2 \alpha-1)}\left(L_{1}+(3-\alpha) L_{2}\right)$.

From the fact that $\frac{5 \alpha+2}{4}>\frac{3 \alpha}{2}$, by (3.25) and (3.26), we immediately have that

$$
\|A u-A v\|_{1} \leq D\|u-v\|_{1}
$$

As $D<1, A$ is a contraction mapping. So, by the contraction mapping principle, $A$ has a unique fixed point $\bar{u}$. That is, $\bar{u}$ is the unique solution of BVP (1.1)-(1.2).

We give another result in this paper as follows.

Theorem 3.2 If $\left(\mathrm{H}_{1}\right),\left(\mathrm{H}_{4}\right)$ hold, then BVP (1.1)-(1.2) has at least one solution.

Proof First, by Lemma 3.2, we know that $A: X_{1} \rightarrow X_{1}$ is completely continuous.

Let $p_{0}=\frac{(5 \alpha+2) \Gamma(\alpha-1)}{4 \Gamma(2 \alpha-1)}, e_{0}=\frac{3 \alpha \Gamma(\alpha-1)}{2 \Gamma(2 \alpha-1)}, q_{0}=\sup _{t \in(0,1]} t^{2-\alpha} \phi(t), d_{0}=p_{0} q_{0}, d_{1}=e_{0} q_{0}$, and $\theta=$ $\max \left\{\theta_{1}, \theta_{2}\right\}$. Put $D=p_{0}\left(N_{1}+(3-\alpha) N_{2}\right), G=e_{0}\left(N_{1}+(3-\alpha) N_{2}\right)$.

Take $R>\max \left\{1,2 d_{0}, 2 d_{1},(2 D)^{\frac{1}{1-\theta}},(2 G)^{\frac{1}{1-\theta}}\right\}$, and set $\Omega=\left\{u:\|u\|_{1}<R\right\}$. Now, we prove that

$$
\|A u\|_{1} \leq\|u\|_{1}, \quad \forall u \in \partial \Omega
$$

In fact, for any $u \in \partial \Omega$, by $\left(\mathrm{H}_{4}\right)$ and (2.25) together with (3.7), we have

$$
\begin{aligned}
\left|t^{2-\alpha} A u(t)\right| \leq & t \int_{0}^{1} G_{1}(s)|F u(s)| d s+\int_{0}^{1}\left|G_{2}(s)\right| \cdot|F u(s)| d s \\
& +\frac{t^{2-\alpha}}{\Gamma(\alpha)} \int_{0}^{t}(t-s)^{\alpha-1}|F u(s)| d s \\
\leq & {\left[\int_{0}^{1} G_{1}(s) s^{\alpha-2} d s+\int_{0}^{1}\left|G_{2}(s)\right| s^{\alpha-2} d s+\frac{1}{\Gamma(\alpha)} \int_{0}^{1}(1-s)^{\alpha-1} s^{\alpha-2} d s\right] } \\
& \cdot\left(\sup _{t \in(0,1]} t^{2-\alpha} \phi(t)+N_{1}\|u\|_{1}^{\theta_{1}}+N_{2}(3-\alpha)\|u\|_{1}^{\theta_{2}}\right) \\
\leq & \frac{(5 \alpha+2) \Gamma(\alpha-1)}{4 \Gamma(2 \alpha-1)}\left(\sup _{t \in(0,1]} t^{2-\alpha} \phi(t)+N_{1}\|u\|_{1}^{\theta_{1}}+N_{2}(3-\alpha)\|u\|_{1}^{\theta_{2}}\right)
\end{aligned}
$$




$$
\begin{aligned}
& =p_{0}\left(q_{0}+N_{1} R^{\theta_{1}}+N_{2}(3-\alpha) R^{\theta_{2}}\right) \\
& <p_{0}\left(q_{0}+\left(N_{1}+(3-\alpha) N_{2}\right) R^{\theta}\right) \\
& =d_{0}+D R^{\theta}<R
\end{aligned}
$$

because

$$
\int_{0}^{1} G_{1}(s) s^{\alpha-2} d s+\int_{0}^{1}\left|G_{1}(s)\right| s^{\alpha-2} d s+\frac{1}{\Gamma(\alpha)} \int_{0}^{1}(1-s)^{\alpha-1} s^{\alpha-2} d s<p_{0},
$$

noting that (2.28), (2.29) and keeping in mind the choice of $R$ and $\|u\|_{1}=R$.

Thus,

$$
\sup _{t \in(0,1]}\left|t^{2-\alpha} A u(t)\right| \leq R
$$

Similarly, by $\left(\mathrm{H}_{4}\right)$ and (2.26) together with (3.7), we have

$$
\begin{aligned}
\left|\left(t^{2-\alpha} A u(t)\right)^{\prime}\right| \leq & \int_{0}^{1} G_{1}(s)|F u(s)| d s+\frac{2-\alpha}{\Gamma(\alpha)} t^{1-\alpha} \int_{0}^{t}(t-s)^{\alpha-1}|F u(s)| d s \\
& +\frac{1}{\Gamma(\alpha-1)} t^{2-\alpha} \int_{0}^{t}(t-s)^{\alpha-2}|F u(s)| d s \\
\leq & \left(\int_{0}^{1} G_{1}(s) s^{\alpha-2} d s+\frac{2-\alpha}{\Gamma(\alpha)} t^{1-\alpha} \int_{0}^{t}(t-s)^{\alpha-1} s^{\alpha-2} d s\right. \\
& \left.+\frac{1}{\Gamma(\alpha-1)} t^{2-\alpha} \int_{0}^{t}(t-s)^{\alpha-2} s^{\alpha-2} d s\right) \\
& \cdot\left(q_{0}+N_{1} R^{\theta_{1}}+N_{2}(3-\alpha) R^{\theta_{2}}\right) \\
= & \left(\frac{\alpha \Gamma(\alpha-1)}{2 \Gamma(2 \alpha-1)}+\frac{(2-\alpha) \Gamma(\alpha-1)}{\Gamma(2 \alpha-1)} t^{\alpha-1}+\frac{\Gamma(\alpha-1)}{\Gamma(2 \alpha-2)} t^{\alpha-1}\right) \\
& \cdot\left(q_{0}+N_{1} R^{\theta_{1}}+N_{2}(3-\alpha) R^{\theta_{2}}\right) \\
< & e_{0}\left(q_{0}+N_{1} R^{\theta_{1}}+N_{2}(3-\alpha) R^{\theta_{2}}\right) \\
< & e_{0}\left(q_{0}+\left(N_{1}+(3-\alpha) N_{2}\right) R^{\theta}\right) \\
= & d_{1}+G R^{\theta}<\left(\frac{1}{2}+G R^{\theta-1}\right) R<R
\end{aligned}
$$

noting that (2.28), (2.29), (2.31) and the choice of $R$ as well as $\|u\|_{1}=R$.

Thus,

$$
\sup _{t \in(0,1]}\left|\left(t^{2-\alpha} A u(t)\right)^{\prime}\right| \leq R
$$

Summing up (3.28) and (3.29), we have that $\|A u\|_{1} \leq R$. That is, $\|A u\|_{1} \leq\|u\|_{1}$ because $\|u\|_{1}=R$. So, the relation (3.27) holds. As a consequence of Lemma 2.6, the operator $A$ has at least one point $\bar{u}$. That is, $\bar{u}$ is a solution of BVP (1.1)-(1.2).

Example 3.1 Consider the following anti-periodic boundary value problem:

$$
\left\{\begin{array}{l}
D_{0+}^{\frac{3}{2}} u(t)=a t^{\beta}+\frac{1}{10 \sqrt{\pi}(1+t)} \frac{|u(t)|}{1+|u(t)|}-\frac{t}{5 \sqrt{\pi}} \ln \left(1+\left(u^{\prime}(t)\right)^{2}\right), \\
t^{\frac{1}{2}} u(t)_{\mid t \rightarrow 0^{+}}=-t^{\frac{1}{2}} u(t)_{\mid t=1}, \quad\left(t^{\frac{1}{2}} u(t)\right)_{\mid t \rightarrow 0^{+}}^{\prime}=-\left(t^{\frac{1}{2}} u(t)\right)_{\mid t=1}^{\prime},
\end{array}\right.
$$


where $\alpha=\frac{3}{2}, \beta \geq-\frac{1}{2}$ and $a \in \mathbb{R}$. Clearly, the function $f(t, x, y)=a t^{\beta}+\frac{1}{10 \sqrt{\pi}(t+1)} \frac{|x|}{1+|x|}-$ $\frac{t}{5 \sqrt{\pi}} \ln \left(1+y^{2}\right)$ satisfies $\left|f\left(t, x_{2}, y_{2}\right)-f\left(t, x_{1}, y_{1}\right)\right| \leq \frac{1}{10 \sqrt{\pi}}\left|x_{2}-x_{1}\right|+\frac{t}{5 \sqrt{\pi}}\left|y_{2}-y_{1}\right|$, and $\sup _{t \in(0,1]}\left|t^{2-\alpha} f(t, 0,0)\right|<\infty$. Further, $L_{1}=\frac{1}{10 \sqrt{\pi}}, L_{2}=\frac{1}{5 \sqrt{\pi}}$, and $D=\frac{19}{20}<1$. As a consequence of Theorem 3.1, BVP (3.30) has a unique solution.

Example 3.2 Consider the following anti-periodic boundary value problem:

$$
\left\{\begin{array}{l}
D_{0+}^{\frac{3}{2}} u(t)=\left(1+e^{M t}\right) t^{-\frac{1}{2}}+(\tan t) \sin \frac{u(t)}{1+(\sqrt[3]{u(t)})^{2}}+2(\sin \pi t) \ln \left(1+\left(\sqrt[5]{u^{\prime}(t)}\right)^{2}\right), \\
\quad t \in(0,1), \\
t^{\frac{1}{2}} u(t)_{\mid t \rightarrow 0^{+}}=-t^{\frac{1}{2}} u(t)_{\mid t=1}, \quad\left(t^{\frac{1}{2}} u(t)\right)_{\mid t \rightarrow 0^{+}}^{\prime}=-\left(t^{\frac{1}{2}} u(t)\right)_{\mid t=1}^{\prime},
\end{array}\right.
$$

where $\alpha=\frac{3}{2}, M \in \mathbb{R}$. Clearly, the function $f(t, x, y)=\left(1+e^{M t}\right) t^{-\frac{1}{2}}+(\tan t) \frac{x}{1+(\sqrt[3]{x})^{2}}+$ $2(\sin \pi t) \ln \left(1+(\sqrt[5]{y})^{2}\right)$ satisfies $|f(t, x, y)| \leq \phi(t)+(\tan 1)|x|^{\frac{1}{3}}+2|y|^{\frac{2}{5}}$, where $\phi=\left(1+e^{M t}\right) t^{-\frac{1}{2}} \in$ $X_{0}$, So, all the assumptions of Theorem 3.2 are satisfied. Hence BVP (3.16) has at least one solution.

\section{Competing interests}

The author declares that he has no competing interests.

\section{Acknowledgements}

The author sincerely thanks the anonymous referees for their valuable suggestions and comments which have greatly helped improve this article. Supported by the Natural Science Foundation of Hubei Provincial Education Department (D20102502)

Received: 25 July 2013 Accepted: 11 October 2013 Published: 08 Nov 2013

\section{References}

1. Samko, SG, Kilbas, AA, Marichev, IO: Fractional Integrals and Derivatives Theory and Applications. Gordon \& Breach, Yverdon (1993)

2. Podlubny, I: Fractional Differential Equations. Academic Press, San Diego (1999)

3. Kilbas, AA, Srivastava, HM, Trujillo, Jj: Theory and Applications of Fractional Differential Equations. North-Holland Mathematics Studies, vol. 204. Elsevier, Amsterdam (2006)

4. Lakshmikantham, V, Leela, S, Vasundhara, J: Theory of Fractional Dynamic Systems. Cambridge Academic Publishers, Cambridge (2009)

5. Diethelm, K: The Analysis of Fractional Differential Equations. Springer, New York (2010)

6. Agarwal, RP, Benchohra, N, Hamani, S: A survey on existence result for boundary value problems of nonlinear fractional differential equations and inclusions. Acta Appl. Math. 109, 973-1033 (2010)

7. Agarwal, RP, Ntouyas, SK, Ahmad, B, Alhothuali, M: Existence of solutions for integro-differential equations of fractional order with nonlocal three-point fractional boundary conditions. Adv. Differ. Equ. 2013, Article ID 128 (2013)

8. Liu, Y, Ahmad, B, Agarwal, RP: Existence of solutions for a coupled system of nonlinear fractional differential equations with fractional boundary conditions on the half-line. Adv. Differ. Equ. 2013, Article ID 46 (2013)

9. Chai, G: Existence results for boundary value problems of nonlinear fractional differential equations. Comput. Math. Appl. 62, 2374-2382 (2011)

10. Chai, G: Existence results of positive solutions for boundary value problems of fractional differential equations. Bound. Value Probl. 2013, Article ID 109 (2013)

11. Chai, G: Positive solutions for boundary value problem of fractional differential equation with $p$-Laplacian operator. Bound. Value Probl. 2012, Article ID 18 (2012)

12. Liu, X, Jia, M, Ge, W: Multiple solutions of a $p$-Laplacian model involving a fractional derivative. Adv. Differ. Equ. 2013, Article ID 126 (2013)

13. Ahmad, B, Nieto, JJ: Sequential fractional differential equations with three-point boundary conditions. Comput. Math. Appl. 64, 3046-3052 (2012)

14. Verma, AK, Agarwal, RP: Upper and lower solutions method for regular singular differential equations with quasi-derivative boundary conditions. Commun. Nonlinear Sci. Numer. Simul. 17, 4551-4558 (2012)

15. Vong, SW: Positive solutions of singular fractional differential equations with integral boundary conditions. Math. Comput. Model. 57, 1053-1059 (2013)

16. Henderson, J, Luca, R: Existence of positive solutions for a system of higher-order multi-point boundary value problems. Appl. Math. Comput. 219, 3709-3720 (2012)

17. Baleanu, D, Mohammadi, H, Rezapour, S: On a nonlinear fractional differential equation on partially ordered metric spaces. Adv. Differ. Equ. 2013, Article ID 83 (2013)

18. Liu, Y, Ahmad, B, Agarwal, RP: Existence of solutions for a coupled system of nonlinear fractional differential equations with fractional boundary conditions on the half-line. Adv. Differ. Equ. 2013, Article ID 46 (2013) 
19. Bai, Z, Sun, W: Existence and multiplicity of positive solution for singular fractional boundary value problems. Comput. Math. Appl. 63, 1369-1381 (2012)

20. Ahmad, B, Ntouyas, SK: Existence results for nonlocal boundary value problems of fractional differential equations and inclusions with strip conditions. Bound. Value Probl. 2012, Article ID 55 (2012)

21. Jankowski, T: Fractional equations of Volterra type involving a Riemann-Liouville derivative. Appl. Math. Lett. 26, 344-350 (2013)

22. Ahmad, B, Nieto, JJ: Riemann-Liouville fractional differential equations with fractional boundary conditions. Fixed Point Theory 13, 329-336 (2012)

23. Ahmad, B, Nieto, JJ: Anti-periodic fractional boundary value problems with nonlinear term depending on lower order derivative. Fract. Calc. Appl. Anal. 15, 451-462 (2012)

24. Chai, G: Existence results for anti-periodic boundary value problems of fractional differential equations. Adv. Differ Equ. 2013, Article ID 53 (2013)

25. Agarwal, RP, Ahmad, B: Existence theory for anti-periodic boundary value problems of fractional differential equations and inclusions. Comput. Math. Appl. 62, 1200-1214 (2011)

26. Wang, G, Ahmad, B, Zhang, L: Impulsive anti-periodic boundary value problems for nonlinear differential equations of fractional order. Nonlinear Anal. 74, 792-804 (2011)

27. Ahmad, B, Nieto, JJ: Anti-periodic fractional boundary value problems. Comput. Math. Appl. 62, 1150-1156 (2011)

28. Wang, F, Liu, Z: Anti-periodic fractional boundary value problems for nonlinear differential equations of fractional order. Adv. Differ. Equ. 2012, Article ID 116 (2012)

29. Alsaedi, A, Ahmad, B, Assolami, A: On antiperiodic boundary value problems for higher-order fractional differential equations. Abstr. Appl. Anal. 2012, Article ID 325984 (2012)

30. Chen, T, Liu, W: An anti-periodic boundary value problems for fractional differential equations with $p$-Laplacian operator. Appl. Math. Lett. 25, 1671-1675 (2012)

31. Li, X, Chen, F, Li, X: Generalized anti-periodic boundary value problems of impulsive fractional differential equations. Commun. Nonlinear Sci. Numer. Simul. 18, 28-41 (2013)

32. Bai, C: Impulsive periodic boundary value problems for fractional differential equation involving Riemann-Liouville sequential fractional derivative. J. Math. Anal. Appl. 384, 211-231 (2011)

33. Wang, W, Shen, J: Existence of solutions for anti-periodic boundary value problems. Nonlinear Anal. 70, 598-605 (2009)

34. Bai, Z, Lü, H: Positive solutions for boundary value problem of nonlinear fractional differential equation. J. Math. Anal. Appl. 311, 495-505 (2005)

35. Sun, J: Nonlinear Functional Analysis and its Application. Science Press, Beijing (2008)

10.1186/1687-1847-2013-306

Cite this article as: Chai: Anti-periodic boundary value problems of fractional differential equations with the Riemann-Liouville fractional derivative. Advances in Difference Equations 2013, 2013:306

\section{Submit your manuscript to a SpringerOpen ${ }^{\ominus}$ journal and benefit from:}

- Convenient online submission

- Rigorous peer review

- Immediate publication on acceptance

Open access: articles freely available online

- High visibility within the field

- Retaining the copyright to your article 